the evidence in such cases may need to be monitored, and there may be an argument for a carefully trained and experienced small cadre of specialists to do the work.

The existing procedures have many faults, some of which I highlighted earlier this year (21 May, p 1421). The working party has answered criticisms of delay in dealing with $\mathrm{HM}(61) 112$ procedures by setting indicative time limits which it hopes will keep cases to a maximum of eight months from the establishment of a prima facie case. Other suggestions of a central standing panel of lawyers readily available to act as chairmen and of legal representation on both sides by solicitors rather than barristers are presumably intended to offer a speeding up to the process and a reduction in costs. They may, however, raise questions about quality and efficiency. The appeals procedure is improved by time limits applying to all concerned, the use of a legal assessor or adviser, and the use of a compromise solution only after its acceptability has been established. The appeals procedure will not be available to those dismissed for personal mis- conduct or - for some extraordinary reason - to practitioners with a limited work commitment with health authorities and an important alternative source of income. The penultimate recommendation of early retirement is most welcome. It is a sensible preventive measure that might have come right at the beginning of all the proposals.

Generally this report represents real progress, and I hope that all concerned agree to start formal negotiations. I would like to see rapid implementation followed by extension to other medical grades as soon as possible.

MALCOLM FORSYTHE

Regional Medical Officer,

South East Thames Regional Health Authority,

Bexhill on Sea,

East Sussex TN39 3NG

1 Joint working party of representatives of health departments, NHS, and professions. Disciplinary procedures for hospital and community doctors and dentists. London: Department of Health and Social Security, 1988.

2 Department of Health and Social Security. Health service management. London: DHSS, 1988. ( $\mathrm{HC}(88) 37$.

\section{Health effects of Chernobyl}

\section{Important to measure among evacuees}

On 26 April 1986 the world's most serious accident in a nuclear power station happened at Chernobyl in the Ukraine. The reactor core and parts of the reactor turbine buildings were destroyed, and large amounts of radioactive materials were released into the atmosphere over 10 days. Acute radiation sickness killed 28 people at the reactor site, and more than 100000 people living within a $30 \mathrm{~km}$ radius were evacuated.

The radioactive fallout primarily affected Europe and was unevenly distributed. Short lived iodine-131 and long lived caesium-137 were the main sources of radiation exposure, which resulted from both external radiation and ingestion of contaminated food. The United Nations Scientific Committee on the Effects of Atomic Radiation (UNSCEAR) has recently compiled data on the geographical distribution of radiation doses after the accident. ${ }^{1}$ For the first year the highest doses to people outside the evacuation zone were estimated to be $2 \mathrm{mSv}$ in Byelorussia in the Soviet Union and between 0.6 and $1.3 \mathrm{mSv}$ in southern Switzerland, north east Romania, northern Greece, Bulgaria, Austria, and western Yugoslavia. The total doses (dose commitments) are about two to four times higher. For comparison, the average dose during one year from natural radiation is about $2.4 \mathrm{mSv}$.

The evacuees received much higher doses. The 24000 people living between $3 \mathrm{~km}$ and $15 \mathrm{~km}$ from the plant are calculated to have received average doses from external radiation of about $350-550 \mathrm{mSv} .^{2}$ The rest of the evacuees received $30-60 \mathrm{mSv}$. In regions near Chernobyl but outside the evacuation zone the dose commitment from internal radiation was roughly equal to that from external radiation.

Several types of health effects may occur as a result of the accident. Effects caused by the radiation are of particular interest; they may include birth defects, childhood cancer, and mental retardation among those exposed in utero. With longer follow up other cancers may also appear. Also to be considered are the side effects of the massive programmes of iodine prophylaxis carried out in some populations. Other health effects include anxiety and fear, which have probably affected many people: such effects are difficult to measure but may show up in extreme circumstances as higher rates of induced abortions or lower birth rates.

Generally two different methods may be used to assess the health effects of the accident at Chernobyl. The first approach is to employ risk estimates based on observations of other populations exposed to radiation. This may be done immediately. The second approach is to conduct epidemiological investigations among those exposed to fallout from Chernobyl. This approach may provide important empirical information that may be useful in improving estimates of the risks of radiation. It should be emphasised that adequate data on radiation doses are a prerequisite for both approaches.

UNSCEAR has recently reassessed the risk estimates of radiation in causing cancer. ${ }^{1}$ Studies on the survivors of the bombings in Hiroshima and Nagasaki provide the most imporant basis for these assessments, and mainly as a result of new (lower) estimates of the doses and longer follow up the risk per unit dose has been raised. Depending on the projection model, the present estimates of cancer risks range from $4 \%$ (additive model) to $11 \%$ (multiplicative model) per sievert. For low doses and dose rates a reduction factor of between two and 10 was assumed, implying a risk estimate ranging from 0.4 to $5.5 \%$ per sievert.

These risk estimates and the available data on radiation doses outside the evacuation zone suggest that any effects of Chernobyl on the incidence of cancer would be difficult to detect. If very large population groups are studied-for example, through national registers-statistical power may not be the major problem, but fluctuations in the incidence of a magnitude that could possibly be caused by the radiation would be difficult to interpret because of a lack of knowledge of other factors affecting the incidence.

Studies may sometimes be done for other than purely scientific reasons. Thus despite all the problems several European countries have started epidemiological investigations into the effects of the accident at Chernobyl. ${ }^{2}$ A Swedish 
register based study on pregnancy outcome in areas heavily affected by fallout from Chernobyl showed no effects on rates of spontaneous abortion, low birth weight, perinatal mortality, or specific malformations. ${ }^{3}$ An increased rate of induced abortions and a lower conception rate were, however, seen in the few months after the accident. It is not known whether these changes were related to the accident.

Epidemiological investigations of the people heavily exposed to radiation around Chernobyl will be of great scientific interest. Actions have already been taken to compile a radiation register of more than 100000 people who received the highest doses. They will be followed up both short term and long term. A new institute has been set up in Kiev to coordinate work under the auspices of the Academy of Medical Sciences of the Soviet Union. International cooperation on epidemiological studies after Chernobyl has been initiated by the International Atomic Energy Authority and the World Health Organisation. ${ }^{2}$
We nuust hope that the efforts to carry out well designed epidemiological studies after the accident will be successful. A heavy responsibility lies with authorities and scientists, particularly in the Soviet Union, to make use of the opportunity created by the tragic events at Chernobyl to expand our knowledge on health risks associated with exposure to ionising radiation.

Professor,

GÖRAN PERSHAGEN

Institute of Environmental Medicine,

Karolinska Institute,

S-10401 Stockholm,

Sweden

1 United Nations Scientific Committee on the Effects of Atomic Radiation. Sources, effects and risks of ionizing radiation. Report of the 37 th session with annexes. Vienna: UNSCEAR (in press).

World Health Organisation Regional Office for Europe. Nuclear accidents and epidemiology. Copenhagen: WHO, 1987

Källén B. Pregnancy outcome in Sweden after Chernobyl-a study with central health registries. Stockholm: National Board of Health, 1988.

\section{Peyronie's disease}

\section{Treatment should always restore sexual function}

Even though he was not the first to describe it the name of Francois Gigot de la Peyronie, ${ }^{1}$ surgeon to Louis XV of France, has become synonymous with the enigmatic condition of Peyronie's disease. The disease is characterised by irregular dense plaques of fibrous tissue, usually on the dorsum of the penile shaft, accompanied by distortion or angulation of the erect penis and in the early stages pain on erection. The erectile deformity may interfere with vaginal penetration making coitus difficult or impossible. If the plaque is extensive and associated with fibrosis of the underlying cavernosal erectile tissue it may result in a band like constriction or waisting of the penile shaft, which leads to flaccidity of the corpora distally. Failure of erection in men with Peyronie's disease is usually psychogenic and secondary to pain or performance anxiety. Impotence, however, may be caused by coexistent penile arterial insufficiency, autonomic neuropathy (particularly in diabetic men), or cavernosal venoocclusive incompetence.

The aetiology of Peyronie's disease remains obscure, and it is not possible to predict its natural course in an individual patient at presentation. Although the pain almost always subsides within a few months, the plaque, and hence the degree of erectile deformity, may either resolve, stay the same, or progress further. The uncertainty about the outcome as well as the need for a period of observation must be explained to the patient.

Peyronie's disease can now be evaluated much more objectively using modern technology. The dimensions of the plaque, the presence of calcification within it, and fibrotic encroachment upon the cavernosal spongy tissue can be accurately defined by means of high resolution ultrasonography. ${ }^{3+}$ Penile arterial inflow can be simultaneously measured using a duplex scanner which combines the above imaging modality with a pulsed Doppler system.' Morphological alterations in the cavernosal bodies can be determined also by computed tomography, ${ }^{6}$ nuclear magnetic resonance imaging of the erect penis, ${ }^{7}$ and by cavernosography. ${ }^{8}$ Dynamic infusion cavernosometry ${ }^{9}$ may usefully complement cavernosography if venous leak impotence is suspected. The erectile deformity may be recorded by polaroid photography either at home ${ }^{10}$ or in the outpatient clinic by inducing an erection with an intracavernosal injection of papaverine. ${ }^{.1}$

The variability in the course of the disease makes it difficult to evaluate the treatments used in the initial acute phase. Thus vitamin $\mathrm{E},{ }^{12}$ potassium aminobenzoate,${ }^{13}$ and orgotein ${ }^{1415}$ are of doubtful benefit, and there is no convincing evidence that radiotherapy, ${ }^{16}$ ultrasonic treatment,${ }^{17}$ or steroids injected directly into the plaque ${ }^{1819}$ influence the disease apart from perhaps hastening the resolution of pain. The therapeutic potential of purified clostridial collagenase injected into the plaque is presently undergoing experimental and clinical trials in the United States. ${ }^{20}$ As yet the preparation is neither available nor licensed in Britain.

The usefulness of surgery in Peyronie's disease is well defined. If the patient is unable to penetrate because of curvature of the penis then it may be straightened. In Nesbit's operation single or multiple ellipses or diamond shaped segments of normal tunica albuginea are excised from the corpora cavernosa opposite the point of maximum curvature; suturing then straightens the penis, albeit at the expense of length. ${ }^{2122}$ This operation is simpler, less time consuming, and more predictable than attempts at excising the plaques and replacing the defects with a dermal graft or other substitutes. ${ }^{23}$ If the patient has organic impotence then a semirigid penile prosthesis may be inserted with or without excision or incision of the plaques..$^{2+}$ Alternatively, intracavernosal self injections of papaverine may be employed, if necessary, as an adjunct to corrective surgery. In patients with psychogenic impotence self injection may be required only short term. In conclusion, there is no reason why Peyronie's disease should end the sexual function of any man.

J C GINGELL Consultant Urologist K M DESAI Southmead General Hospital, Senior Registrar in Urology Bristol BS10 5NB

1 Peyronie F de la. Sur quelques obstacles qui s'opposent à l'éjaculation naturelle de la semence. Memoires de L'Academie de Chirurgie 1743;1:318-33.



